\title{
VISION WITHOUT INVERSION OF THE RETINAL IMAGE. ${ }^{1}$
}

\author{
BY PROFESSOR G. M. STRATTON.
}

University of California.

Seventh Day.-In the morning the flow of ideas while I was blindfolded was like that described for the evening before. But $I$ noticed in bathing that the old representation of those parts of my body which I had so frequently seen (at least in their clothing) during the experiment, was decidedly less vivid, the outline more blurred, the color paler, grayer, more ' washed out,' than of the parts which had never come within the limits of the visual field.

Later, with my lenses on, it seemed at first as if the experience was in all respects the same as on the previous day. But when I began to pace rapidly up and down the room, I felt that I was more at home in the scene than ever before. There was perfect reality in my visual surroundings, and I gave myself up to them without reserve and without being conscious of a single note of discord with what I saw. This feeling of complete harmony throughout, lasted as long as I kept my legs either within or near the borders of my field of view. Otherwise the older, inappropriate representation of my body arose at times, but faded, while the new representation revived, as soon as some passing object was seen to enter the region into which the older image of my body extended. The absence of any tactual experiences such as a real body in that position would imply, cast, for the moment, an illusory character over the older form of representation.

To what extent objects in view suggested the idea of other things in harmonious relation with the seen things is best shown by the following cases: As I walked into my bedroom and saw the bedstead, I involuntarily thought of the windows, repre-

1 Concluded. 
senting them in the appropriate direction fixed by the position of the bed. The general outlines of the room, and the more important points of reference, arose in harmony with the new sight-perceptions. But the detailed filling of this outline was far less complete than is usual in my case in normal sight. A large number of important things in the room simply did not arise in my mind until their relation to the field of seen things had been brought home afresh by perception. During the first days of the experiment ideas of objects frequently arose in opposition to the new sight-perception; now they either did not arise at all, or came in the newer form. The idea of the sofa or chair on which I passively sat did still come up in discord with the general experience, together with the dim feeling of my shoulders and of the upper parts of my back. But these were now a comparatively isolated group, and not a vigorous Apperceptions-masse to call up a host of surrounding things in orderly relation to itself.

In regard to movements, the most striking fact was that the extent of the movement now was inappropriate, movements in the wrong direction being comparatively rare in the case of the hands, and even still rarer in the case of the feet. My hands frequently moved too far or not far enough, especially when coming from beyond the visual field to something in sight. In trying to take a friend's hand, extended into the (new) lower portion of my visual field, I put my hand too high. In brushing a speck from my paper in the (new) upper portion of the field I did not move my hand far enough. And in striking with my index finger the outstretched fingers of my other hand the movement was much less accurate when I looked at my hands than when I closed my eyes and depended on motor guidance. The actual distance that my hand moved, in such cases, would, under the normal conditions of sight, doubtless have been appropriate to bring my hand to the desired spot. But an object in what had before been the upper part of the field was now at a shorter distance from my hands than formerly; the movement, under the influence of the habitual interpretation of the visual position, would therefore go too far. And, vice versa, a movement to an object in what had formerly 
been the lower part of the visual field would now fall short of its destination. For the visual position would now require a more extended movement of the arm than formerly, in order to reach it.

When I watched one of my limbs in motion, no involuntary suggestion arose that it was in any other place or moved in any other direction than as sight actually reported it, except that in moving my arm a slightly discordant group of sensations came from my unseen shoulder. If, while looking at the member, I summoned an image of it in its old position, then I could feel the limb there too. But this latter was a relatively weak affair, and cost effort. When I looked away from it, however, I involuntarily felt it in its pre-experimental position, although at the same time conscious of a solicitation to feel it in its new position. This representation of the moving part in terms of the new vision waxed and waned in strength, so that it was sometimes more vivid than the old, and sometimes even completely overshadowed it.

The conflict between the old and the new localization of the parts of my body was shown in several instances. The mistaken visual localization of a contact in the palm of one of my hands, and the sudden reversal of even the touch-localization when I detected by sight the true source of the sensations, occurred as on the preceding day. Somewhat similarly, when I moved a heated iron with my right hand to that border of the visual field just beyond which, according to pre-experimental localization, my left hand would have been lying, I involuntarily felt an anticipatory shrinking in my unseen left hand, as if it were on the point of being burnt; although the iron in my right hand was actually several feet from my left, and was moving away from it. When I put my left hand in sight, or looked at it afresh to make sure where it was, the hot iron caused no premonitory feeling whatever on approaching the visual locality which had before been so suggestive of danger.

Seated by the open fire, I happened to rest my head on my hands in such a way that the fire shone directly on the top of my head. I closed my eyes, and the image of the fire remained true to the recent perception. But soon I noticed that I was 
representing the fire in pre-experimental terms, and I finally discovered that the change was caused by the growing sensations of warmth on the top of my head. My hair and scalp were persistently felt in their older position, no doubt because I never. directly saw them in any other. And the old localization of the fire was the only one consistent with this old localization of the hair and scalp. But by passing my hands rapidly back and forth before my open eyes, ending the movement each time with a touch upon the top of my head, it was not difficult to produce a vivid localization of my scalp in harmony with the new sightperceptions. And with this change the old localization of the fire was suppressed. During the walk in the evening, I enjoyed the beauty of the evening scene, for the first time since the experiment began. Evidently the strangeness and inconvenience of the new relations no longer kept me at such a tension as hitherto.

On removing the glasses, my visual images relapsed into their older form, with a constant interplay and accompaniment, however, of the new.

Eighth day.-Before putting the glasses on, representations of the older sort held sway.

During the morning, after the glasses were in place, I noticed that as far as the unseen portions of my body were concerned, the relation of right and left was, for the most part, a reproduction of the older visual right and left; that is to say, a contact on the right side of the body at some point beyond the reach of sight was felt and visually represented on the (old) visual right side. Occasionally the opposite visual side was suggested, but the sensations were rarely indeed felt there. The case was quite different as regards the seen parts of my body, although even here uncertainly and sudden alteration of reference occurred. The illusion of contact on the opposite hand to the one actually touched, arose as on the two preceding days. I often hesitated which hand was the appropriate one for grasping some object in view, began the movement with the wrong hand and then corrected the mistake. If $I$ was attentive to the new visual representation of some part of my body which was about to be touched, and expected the contact 
there, the contact was felt in the new position and no change of reference occurred. Immediately afterwards there usually arose a sort of tactual after-image on the other visual side. When the original contact was unexpected, the visual image and the tactual localization might simultaneously be both old and new, or might be old alone, with perhaps a merely visual image in the new direction, although without any real reference of the touch-sensations to this image.

Localization of sounds varied, being different when the source of sound was in sight from what it was when this was out of sight, and also in the latter case differing with different directions of attention, or with different suggestions as to the direction from which the sound came. The fire, for instance, sputtered where I saw it. The tapping of my pencil on the arm of my chair seemed without question to issue from the visible pencil. Even when I tapped on the wall to one side, out of sight, if in making the stroke I invariably passed my hand and pencil before my eyes and in the direction of the unseen part of the wall, and attempted to picture the contact in harmony with this movement, I actually heard the sound come from the new visual direction, although not with full and unequivocal localization. There was a strong temptation to localize the sound on the other side also. And this rival localization rose into full life the instant $I$ ceased to keep before me the image of the pencil striking on the new visual side.

The influence of the suggestion coming from recent and repeated movements before the eyes was likewise apparent in localizing parts of my body which could not be brought into the visual field. Thus the involuntary inattentive localization of my forehead and hair was the old localization lasting from preexperimental sight. But a series of visible movements of my hand to my hair, together with fixed attention on the goal of these movements, made the sensations of touch temporarily come, without difficulty, from this new direction. Sensations of contact on the lips, however, were not so readily dislodged from their old position. In eating at table, the movements of my hands and of pieces of food across the visual field, constantly suggested that my mouth must lie between the line of 
sight and the new position of my legs. But the actual contact on my lips instantly dispelled this suggestion and located my mouth definitely and indubitably on the other side of the line of sight. The place of the actual contact and that of the merely suggested contact were thus in striking contrast. But when I did my best to visualize my lips in the direction of the suggested contact and strained my attention in this direction, the actual contact did not dissipate this image or carry it to the old position of my mouth, but the touch-sensations seemed to come from the new direction. Without such a willful visualization and strain of attention the actual contact always reversed the involuntary suggestion coming from the visible movements toward the new position of my mouth. Even when my forehead and hair temporarily seemed to lie on the (new) upper side of the line of sight, this did not prevent my mouth from being felt on the same side. But the new localization of forehead and scalp undoubtedly had a tendency to drive the mouth out of its old localization; for I found that less effort of attention and visualization was required to make the tactual sensations of the lips come from the new position, when the top of the head had already been carried over to its new position, No doubt there was a disturbing incongruity in having both my mouth and the top of my head on the same side of the line of sight; consequently the re-localization of one tended to carry the other to the opposite side of the visual line.

In other cases the re-localization of bodily parts that were beyond the reach of sight was brought about by the suggestive influence of such movements as I have just described, without any voluntary attention or visualization of the parts whatever. As $I$ rocked in my chair, I found that by throwing my arms up through the field of sight into the visual region in which my shoulders, according to the old experience, were wont to be localized, the repeated feeling of the unimpeded motion of $\mathrm{my}$ hands through this region destroyed the old representation of my shoulders and back, and gave them a localization in harmony with the new visual experience, except that (as I noticed) my head seemed too deep-set in my shoulders-in fact, seemed buried in them almost up to my ears. 
The harmonization of the new experience and the suppression or subordination of insistent remnants of the old were always apparent during active operations in the visual surroundings, as has been described for several of the preceding days. While I sat passively the old localization of unseen parts of my body often came back, or perhaps was the usual form in which they appeared. But the instant I began to rock my chair the new position of these parts came prominently forward, and, except in the case of my shoulders and back, readily felt more real than the old. And in walking, when hands and feet rhythmically made their appearance in the visual field, the old representation, except perhaps for some faint inharmonious sensations in the back, was fully expelled without employing any device of will or of attention whatever. The attempt to represent my body in its older form or position ended in a faint, lifeless outline, deficient, as far as I could make out, in those parts which (in a different direction, of course) were actually in sight. The sight of these parts made it impossible to represent them in harmony with the older experience. If in walking I allowed my feet to remain outside the field of view and they relapsed into their older localization, they returned, although still unseen, to their new position as soon as I approached a step or other slight obstacle on the floor.

As long as the new localization of my body was vivid, the general experience was harmonious, and everything was right side up. But when, for any of the reasons already given-an involuntary lapse into the older memory-materials, or a willful recall of these older forms-the pre-experimental localization of my body was prominently in mind, then as I looked out on the scene before me the scene was involuntarily taken as the standard of right directions, and my body was felt to be in an inharmonious position with reference to the rest. I seemed to be viewing the scene from an inverted body.

When the time came for removing the glasses at the close of the experiment, I thought it best to preserve as nearly as possible the size of visual field to which I had now grown accustomed; so that any results observed might be clearly due solely to the 
reversion of my visual objects and not to a sudden widening of the visual field. Instead, therefore, of removing the plastercast from my face, I closed my eyes and had an assistant slip out the brass tube which held the lenses, and insert in its place an empty black-lined paper tube that gave about the same range of vision. On opening my eyes, the scene had a strange familiarity. The visual arrangement was immediately recognized as the old one of pre-experimental days; yet the reversal of everything from the order to which I had grown accustomed during the past week, gave the scene a surprising, bewildering air which lasted for several hours. It was hardly the feeling, though, that things were upside down.

When I turned my body or my head, objects seemed to sweep before me as if they themselves were suddenly in motion. The 'swinging of the scene,' observed so continously during the first days of the experiment, had thus returned with great vividness. It rapidly lost this force, however, so that at the end of an hour the motion was decidedly less marked. But it was noticeable the rest of the day, and in a slight degree even the next morning.

Movements which would have been appropriate to the visual arrangement during the experiment, were now repeatedly performed after this arrangement had been reversed. In walking toward some obstacle on the floor of the room-a chair, for instance-I turned the wrong way in trying to avoid it; so that $I$ frequently either ran into things in the very effort to go around them, or else hesitated, for the moment, bewildered what I should do. I found myself more than once at a loss which hand I ought to use to grasp the door-handle at my side. And of two doors, side by side, leading to different rooms, I was on the point of opening the wrong one, when a difference in the metal work of the locks made me aware of my mistake. On approaching the stairs, I stepped up when I was nearly a foot too far away. And in writing my notes at this time, I continually made the wrong movement of my head in attempting to keep the centre of my visual field somewhere near the point where I was writing. I moved my head upward when it should have gone downward; I moved it to the left when it should have 
gone to the right. And this to such a degree as to be a serious disturbance. While walking, there were distinct signs of vertigo and also the depression in the upper abdominal region, noticed during the earlier days of the experiment. The feeling that the floor and other visual objects were swaying, in addition to the symptoms just mentioned, made my walking seem giddy and uncontrollable. No distinct errors in localizing parts of my body occurred; I was more than once surprised, however, to see my hands enter the visual field from the old lower side.

Objects in the room, at a distance of ten or twelve feet from me, seemed to have lost their old levels and to be much higher than they were either during the experiment or before the experiment. The floor no longer seemed level, but appeared to slope up and away from me, at an angle of perhaps five degrees. The windows and other prominent objects seemed also too high. This strange aspect of things lasted (as did also the swinging of the scene, the feeling of giddiness, and certain inappropriate movements) after the plaster cast had been removed and the normal compass of the visual field was restored. In the dim light of the next morning, the upward slope of the floor and the unusual position of the windows were distinctly noticeable.

It is clear, from the foregoing narrative, that our total system of visual objects is a comparatively stable structure, not to be set aside or transformed by some few experiences which do not accord with its general plan of arrangement. It might perhaps have been supposed beforehand that if one's visual perceptions were changed, as in the present experiment, the visual ideas of things would without resistance conform to the new visual experiences. The results show, however, that the harmony comes only after a tedious course of adjustment to the new conditions, and that the visual system has to be built anew, growing from an isolated group of perceptions. The older visual representations for the most part have to be suppressed rather than reformed.

Why then do the old visual ideas persist in their old form, and not come immediately into accord with the new perceptions? 
If their position were merely relative to the sight-perceptions, they undoubtedly would come into harmony with these perceptions, at least after the first moments of dismay were past. But the fact that the ideas can for some time refuse spatially to conform to the new experience, shows that their position and direction is fixed with reference to something other than the immediate perceptions of sight. What is it which caused the older visual images to preserve a spatial arrangement whose lines of direction were opposed to those of the actual field of view?

To say that the older visual directions persisted because the older tactual directions remained in force, is certainly no sufficient answer unless we can show that visual direction is dependent on tactual direction. But the preceding narrative furnishes strong evidence against such a view. If there is any dependence either way (which I doubt), the evidence seems to favor the primacy of sight.

However that may be, the facts in the present case are more accurately described when we say that the discord was not between tactual directions and visual directions, but between the visual directions suggested by touch and the visual directions given in the actual sight. The real question then is : Why did touch-perceptions so persistently suggest visual images whose positions and directions were in discord with the actual scene? The answer is found, I think, in the familiar doctrine of 'local signs' in touch and in sight, and in the farther assumption that a system of correspondence exists whereby a sign in one sense comes to be connected with and to suggest a particular sign in the other sense.

In the organized experience, a perception in one sensory field not only has in it that peculiar qualitative or intensive character which is its own 'local sign,' but, through this local sign, suggests in the other sensory field the local sign which is most intimately associated with the first. A perception in one sensory field suggests, therefore, in terms of the other sense an image in that place whose local sign is most strongly associated with the local sign of the original perception. According to this view, the local signs of sight correspond to the signs of touch, and vice versa; so that each member in this system of corresponding 
signs has its particular correlate in the other sensory field. The correspondence here indicated, does not, however, consist in any spatial or qualitative identity or even similarity of the particular signs which correspond, but only in the fact that both have come to mean the same thing. They have occurred in connection with disparate sensory perceptions whose times of appearing and whose 'curve' of change have been so continuously and repeatedly identical that the perceptions themselves come, in time, to be referred to the same source, or, in other words, give the perception to the same object. The perceptions of the two senses are thus identified; and, at the same time, the disparate local signs (in the different senses) which are simultaneously aroused in the perception of the one object come to have the same spatial meaning. This correspondence of local signs is no doubt an important condition for our perceiving one and the same thing in different sensory fields. And the persistence of this correspondence between the signs, when once the power of mutual suggestion has become established, is the explanation of the fact that during my experiment the translations of touch-perceptions into terms of sight continued so long in contradiction to the actual visual experience; and that, on the other hand, the visual perceptions so long suggested tactual or motor images not in accord with the tactual or motor perceptions.

For, whatever the local signs of vision may be-whether differences in the qualitative or intensive character of the muscular sensations, or differences of sensation connected with different parts of the retina, or intricate combinations of both of these materials-the reversion of the retinal image would so alter the conditions of sight that the tactual perception of an object and the simultaneous sight of the same object would no longer call into play the pair of local signs which hitherto had had the same spatial meaning, but a pair of signs which had come to have opposed spatial meanings. Suppose, for illustration, that any two tactual local signs, $a$ and $b$, have in my nor-

1 For convenience sake let us speak of the signs as though they could be simple. Of course they are really complexes of sensations from joints and muscles and skin. Similarly of the visual signs. 
mal experience the same meaning as the two visual local signs $m$ and $n$, respectively. A single object which is both seen and touched will arouse the synonymous signs $a$ and $m$, or subsequently the pair $b$ and $n$, supposing that the object changes its place. If I merely touch the object, in such a place that $a$ is aroused, this suggests by associative correspondence the visual sign $m$; and when subsequently I look at the object, $m$ is actually aroused, and the place of the visual object is seen to be identical with the visual place suggested by touch. Touch in this case suggests a visual experience which the visual perception confirms. Likewise a visual perception whose local sign was $n$ would suggest, and afterwards be followed by, a tactual experience whose local sign was $b$. And in these cases the spatial character of the perceptions by the different senses would be harmonious.

But suppose, now, that the retinal image is changed, as in the experiment. An object which arouses the tactual sign $a$ will no longer give a visual experience containing the sign $m$, but will give one containing, say, $n$. And the visual experience containing the local sign $m$ is no longer accompanied by a tactual experience containing $a$, but by one containing, say, $b$; and vice versa. By the long previous experience, however, a touch-perception containing the sign $a$ has come to suggest a visual experience containing the sign $m$, and will consequently continue for some time to suggest such a visual experience. But the actual sight of the object will show it in a different place from what touch suggested ; for the visual experience will now actually contain $n$ and not $m$. And likewise this visual experience whose local sign is $n$ will for some time continue to mean a tactual experience whose sign is $b$, in a different locality from the real touch of the object, which now contains the sign $a$. Each sense will in this way suggest experiences which the actual perceptions of the other sense will contradict. Thus touch and sight will be in mutual discord.

According to the view here presented, this discord will continue as long as the local sign $a$ suggests the local sign $m$, and vice versa. But when $a$, by repeated connection, suggests only the visual position implied in the local sign $n$, and this latter in 
turn means only the touch locality whose sign is $a$; and when $m$ and $b$ have come to have an identical meaning, or are in correspondence; then the total experience will again be harmonious. Each sense would then suggest only what the other sense would confirm. We would see things where we felt them to be, and we would feel them where we saw them to be. But until this reharmonization has been brought about, visual ideas in the older form will continue to arise at the suggestion of tactual experiences, and there will be discord between the things in sight and the wider system of visual representations. The persistence of the old inter-sensory correspondences accounts, therefore, for the long opposition of visual ideas and visual perceptions, during the experiment.

We are now enabled also to see what the harmony between touch and sight really is. The experiment clearly shows that an object need not appear in any particular position in the visual field in order to admit of a union or identification of the tactual and visual perceptions of the object. The visual position which any tactual experience suggests - the visual place in which we ' feel' that an object is-is determined, not by some fundamental and immutable relation of tactual and visual 'spaces,' but by the mere fact that we have constantly seen the object there when we have had that particular touch-experience. If this particular touch-experience were the uniform and exclusive accompaniment of a visual object in some different visual position, the two sensory reports would mean the same thing, and the places of their object would be identical. Of course, the harmony of touch and sight also implies that visual appearances have the same relations to one another as tactual appearances have to one another; so that a given object in sight must have the same spatial relation to the rest of my visual world as the accompanying touch-object has with respect to the rest of my tactual world. But this harmony does not require that the visual manifestation of a tactual object should be just here and not there, or in this direction and not in that.

The inverted position of the retinal image is, therefore, not essential to 'upright vision,' for it is not essential to a har-: mony between touch and sight, which, in the final analysis, is 
the real meaning of upright vision. For some visual objects may be inverted with respect to other visual objects, but the whole system of visual objects can never by itself be either inverted or upright. It could be inverted or upright only with respect to certain non-visual experiences with which I might compare my visual system-in other words, with respect to my tactual or motor perceptions.

The reharmonizing of touch and sight, in the experiment, consisted therefore of a double work. Visual objects and ideas, which were at first isolated, had to become a system whose parts had the same relations among themselves as the parts of the tactual system, or of the older visual system. Not until the construction of a visual system enveloping and supplementing the actual visual field, would sight have something corresponding to the touch-system brought over undisturbed from the older experience. But the completion of this work was dependent on the progress of the second work, namely the perfecting or entire reconstruction of the process of translating from sight into touch and from touch into sight. Until this reconstruction was complete, each sense would suggest experiences of the other sense which this other sense would flatly contradict. Their reports would therefore necessarily seem discordant. But the restoration of harmony between the perceptions of sight and those of touch was in no wise a process of changing the absolute position of tactual objects so as to make it identical with the place of the visual objects; no more than it was an alteration of the visual position into accord with the tactual. Nor was it a process of changing the relative position of tactual objects with respect to visual objects; but it was a process of making a new visual position seem the only natural place for the visual counterpart of a given tactual experience to appear in; and similarly in regard to new tactual positions for the tactual accompaniment of given visual experiences. New associations had to develop, and new forms of expectation had to arise; in a word, new correspondences had to be brought about. But the tactual perceptions, as such, never changed their place. They simply got a new visual translation.

The especial obstinacy of the old representation of the body 
requires no extended comment. It is what we would expect when the cause of the persistence of the older images in general is understood. If visual suggestion from touch, based on the pre-experimental set of correspondences between touch and sight, was the chief cause of the persistent return of the older images, then of course the conditions were peculiarly favorable for a continuance of the old visual representation of the body. For in this case touch was ever-present; and moreover the body was seen only in part. Head, neck, shoulders, and the upper part of the trunk, could not be directly seen at all. Shadows, reflections, etc., had some influence in bringing to mind the new visual place of these parts; but such indirect agents lacked the force of direct and continued perception. So that the possibility of bringing about new correspondences was confined, for the most part, to my arms and legs. But there is, doubtless, a solidarity of the body, and when so large a part could not be reached by the new experience, the rest also was affected but little. The body hung together as a unit, and refused to go with the new, unless all of it could go.

In the daily experience during the experiment, localization of parts of the body to one side (right or left) of the visual field of representation was more persistently in discord with the new visual experience, than was the vertical localization of these parts. The explanation of this is found, I think, in the fact that both tactual and visual differences in the body are much more striking at different levels of the body (passing up and down) than on different sides of the body. I frequently saw one of my hands and took it for the other, but of course I never looked at my foot and thought it was my hand. So, too, I erroneously localized in one of my hands an object which was in contact with the other hand, but I never localized in my foot a contact that was really on my hand, nor vice versa. The experience itself was thus, as far as lateral relations were concerned, comparatively ambiguous, but not ambiguous at all with respect to vertical relations. When, through the touchexperience, a visual image was called up in the old lateral relations, this image was not so violently in discord with the new visual experience, as a mistake in vertical reference would have 
been. For the image of my right arm or leg would also serve fairly well for that of my left. And in actual sight nearly the same objects were seen now on one side and now on the other. This hindered a fixed association of a particular image with a particular visual side, such that when a contact occurred the part of the body which it suggested must be referred to this side and not to that, if the contract were to fit into the visual total at all. The fact that the new visual experience was tolerant of a localization on either side almost indifferently was therefore favorable to a continuance of the old lateral localization. But the new vision unequivocally pronounced against an error in vertical localization; the uniform contradiction tended therefore to break up the old suggestions, and to build anew the vertical system more rapidly than the lateral.

It is not improbable that the persistence of the old lateral localization of the body was the main cause of the relative permanence of the old localization of sounds. For, vertically, wide changes in localization of sounds readily came by spontaneous suggestion; but not so the lateral changes. These came rarely except by strong effort of attention and voluntary vizualization. The chief basis for determining the side from which a sound comes-the relative intensity of the sound in the two ears-would lead us to expect exactly this result as long as the two sides of the body were involuntarily represented as of old. If a sound was localized with reference to a particular ear or side of my head, then it would be localized in the old way as long as these were localized in the old way; and not until the localization of the two ears or sides of the head was transposed into harmony with the new experience would the auditory localization, at least in its lateral aspects, come into harmony with that experience. The cases in which the lateral localization of sound did accord with the new sight were no doubt due to a momentary strengthening of the influence of visual suggestion to such a degree that the usual dominant factor in lateral localization became subordinate. The fact that the sound of my footsteps conformed to the tactual and visual localization of my feet, and that in general the sight of the sound's place of origin carried with it the localization of the sound, shows to what an extent our auditory localization is influenced by suggestion. 
It was repeatedly noticed in the course of the experiment that the total experience was much more harmonious during active movements of my body than when I inactively looked out upon the scene. This becomes intelligible when one sees how such movements gave additional vivacity to the new visual experience and to all that was in harmony with it, and tended to suppress those images of the body which did not accord with the new relations. The movements of my arms and legs into and through the visual field emphasized their new visual position more than their motionless appearance would have done. They caught and held the attention, and by the vigor of their appearance suggested the rest of the body in harmony with themselves. Moreover the movements of the field of view, when I nodded my head, or moved up and down in walking, or rocked in my chair, were such as harmonized with the movement of my body only when my body was thought of as in its new visual position. Otherwise the objects passed through the field of view in the wrong direction, - in a direction which the felt movement of my head or body did not at all explain. And, finally, the new localization of the body was the only one which was practically important when the visible environment had to be actively encountered. My actions could be guided, not by keeping in mind the pre-experimental localization of the body and noticing its relation to objects in sight, but only by accepting the new position of my body as real and constantly watching its relation to surrounding things. The scene itself became more my own by acting upon it, and this action reacted to bring the representation of my body into harmonious relation to the scene. As a result of these various influences, the whole experience was cleared of inner discord to a degree seldom if ever attained during a time of repose.

At the close of the experiment, after the lenses had been removed, windows and other prominent objects, as the narrative recounts, seemed too high. This was puzzling enough until I discovered that, when my apparatus was on my face, objects in the centre of the field of view were slightly lower than when seen without the lenses. The axis of the cylinder containing the lenses was in fact not exactly the same as the line of sight 
when the eye was directed to the centre of the field. The displacement this caused in the apparent position of things was the less noticeable because of the general transposition of objects by the inverting power of the lenses. But a careful examination showed that the downward displacement at the centre was about equal to the apparent upward displacement on removing the lenses. No exact comparison could be made, for I did not notice this peculiarity of the apparatus until several days after the close of the experiment. So that I had to depend on my inexact recollection of what the extent of the illusion had been. It is, of course, barely possible that the illusion was in some way a direct consequence of reinverting the retinal image. But more probably the general displacement of objects, by reason of the position of the tube, had grown so familiar that their normal position on removing the glasses seemed as much too high as their position during the experiment had been too low.

But to return to the more significant features of the experiment. These are, without doubt, found in the results bearing on the relation between touch and sight, and through them on the interrelation of the senses generally. The experiment makes it clear that the harmony between sight and touch does not depend on the inversion of the retinal image. The spatial identity of tactual and visual objects evidently does not require that there should be a visual transposition of objects or that they should be given some special direction in the visual field. The chief reason for the existence of the projection theory is therefore taken away. Nor, on the other hand, are the visual directions made known to us and determined through our perceiving the 'absolute,' or pure motor, direction of the movements which alter the line of sight. The facts all go to show that the direction of movements of the head or eyes is not judged on purely muscular evidence, independently of the simultaneous changes in vision itself. On the contrary the movements are soon felt as having a direction opposite to that of the objects passing through the visual field. During the experiment, for instance, I often felt my eyes turn toward the sky and away from my feet, although they really turned toward my feet. The felt direction of the movement is therefore relative to the 
direction of the movement of visual objects, and the 'absolute' muscular direction cuts no decisive figure in the perception at all. This will no doubt seem a hard saying to those who have been pinning their faith more and more on the unimpeachable witness of muscular sensations. It certainly makes the eyemovement doctrine of visual directions of little practical assistance for understanding the harmony between sight and touch.

This harmony, as was said, seems rather to be an accord of the ideas suggested in terms of one of the senses, with the perceptions of the same sense. When touch and sight agree, it means that the perceptions of sight are spatially identical with the visual suggestions produced by touch, and that the perceptions of touch spatially identical with the tactual suggestions produced by sight. The doctrine of a correspondence of local signs, stated some pages back, makes it easy to see how such a harmony could grow up ; and, at the same time, how a reharmonization of touch are and sight is possible, whatever may be the position of the retinal image. The view makes provision, therefore, for the special results of the experiment, as well as for the normal course of our experience ; which the current doctrines concerning the interplay of touch and sight seem hardly able to do. 